\title{
Quantum Nonlocality in Phase Space
}

\author{
Konrad Banaszek and Krzysztof Wódkiewiczt \\ Instytut Fizyki Teoretycznej, Uniwersytet Warszawski, Hoża 69, PL-00-681 Warszawa, Poland
}

(September 11, 2018)

We propose an experiment demonstrating the nonlocality of a quantum singlet-like state generated from a single photon incident on a beam splitter. Each of the two spatially separated apparatuses in the setup performs a strongly unbalanced homodyning, employing a single photon counting detector. We show that the correlation functions violating the Bell inequalities in the proposed experiment are given by the joint two-mode $Q$-function and the Wigner function of the optical singlet-like state. This establishes a direct relationship between two intriguing aspects of quantum mechanics: the nonlocality of entangled states and the noncommutativity of quantum observables, which underlies the nonclassical structure of phase space quasidistribution functions.

PACS Number(s): 03.65.Bz, 42.50.Dv

A fundamental step providing a bridge between classical and quantum physics has been given by Wigner in form of a quantum mechanical phase space distribution: the Wigner function [1]. From the pioneering work of Weyl, Wigner and Moyal, it follows that the noncommutativity of quantum observables leads to a real abundance of different in form quantum mechanical phase space quasidistributions. A description of quantum phenomena in terms of the Wigner or the positive- $Q$ quasidistributions, provided a milestone step towards a $c$-number formulation of quantum effects in phase space [2].

Due to Einstein, Podolsky, and Rosen (EPR) [3], followed by the seminal contribution of Bell [4], the meaning of quantum reality and quantum nonlocality has become a central issue of the modern interpretation and understanding of quantum phenomena [5]. Such concepts like entanglement and quantum nonlocality have generated a real flood of theoretical work devoted to various connections of the quantum description with different views or representations of the quantum formalism.

Despite all these theoretical works a direct link between various phase space distributions and the nonlocality of quantum mechanics has been missing. In some works [6] the quantum phase space has been treated as a model for a hidden variable theory, and the incompatibility of quantum mechanics with local theories has been attributed to the nonpositive character of the Wigner function. In this context it has been argued that the original EPR wave function cannot violate the positionmomentum Bell inequality, because the corresponding Wigner function is positive everywhere.

It is the purpose of this Letter to propose an experimental demonstration of nonlocal effects in phase space exhibited by a quantum optical singlet-like state generated from a single photon. The entanglement will be represented by a correlated state of light, which refers to two spatially separated modes of the electromagnetic field. We show that the proposed experiment establishes a direct relationship between quantum nonlocality and the positive phase space $Q$-function, as well as the nonpositive Wigner function. We demonstrate that for a certain class of experiments these two quasiprobability distributions are nonlocal correlation functions violating Bell's inequalities.

In this Letter we propose a realistic photon counting experiment which leads directly to a measurement that is described by the phase space $Q$-function or the Wigner function. We show that these functions are equal to observable joint photon count correlations and as such can be put to test of local realism in form of Bell's inequalities for an entangled single photon. Our approach is different from all the previous discussions involving the relation of quantum nonlocality and the phase space quasiprobability distributions. To the best of our knowledge, no such direct relation between various phase space quasidistributions and the nonlocality of quantum correlations has ever been satisfactorily established.

The link of quantum nonlocality to the $Q$-function is a rather surprising result, since this particular distribution function is positive everywhere, which has been considered as a loss of quantum properties due to simultaneous measurement of canonically conjugated observables.

The setup to demonstrate quantum nonlocality in phase space is presented in Fig. 1. A single photon impinges onto a 50:50 beam splitter. The quantum state written in terms of the outgoing modes, which we will label with $a$ and $b$, is of the form analogous to the singlet state of two spin- $1 / 2$ particles [7]:

$$
|\Psi\rangle=\frac{1}{\sqrt{2}}\left(|1\rangle_{a}|0\rangle_{b}-|0\rangle_{a}|1\rangle_{b}\right) .
$$

Each of the measuring apparatuses consists of a photon counting detector preceded by a beam splitter with the power transmission $T$. The second input port of the beam splitter is fed with a highly excited coherent state $|\gamma\rangle$. As it is known [8], in the limit $T \rightarrow 1$ and $\gamma \rightarrow \infty$, the effect of the beam splitter is described by the displacement operator $\hat{D}(\sqrt{1-T} \gamma)$ with the parameter equal to the amplitude of the reflected part of the coherent state. In the following, we will assume that this limit describes sufficiently well the measuring apparatuses. 
The first type of the measurement we will consider is the test for the presence of photons. This is a more realistic case, as the most efficient detectors available currently for single-photon level light, namely the avalanche photodiodes operating in the Geiger mode, are not capable of resolving the number of photons that triggered the output signal. This type of measurement is described by a pair of two orthogonal projection operators depending on the coherent displacement $\alpha=\sqrt{1-T} \gamma$ :

$$
\begin{aligned}
& \hat{Q}(\alpha)=\hat{D}(\alpha)|0\rangle\langle 0| \hat{D}^{\dagger}(\alpha) \\
& \hat{P}(\alpha)=\hat{D}(\alpha) \sum_{n=1}^{\infty}|n\rangle\langle n| \hat{D}^{\dagger}(\alpha)
\end{aligned}
$$

which satisfy the completeness relation:

$$
\hat{Q}(\alpha)+\hat{P}(\alpha)=\hat{\mathbb{1}} .
$$

In the following, we will use the indices $a$ and $b$ to refer to the two apparatuses.

In contrast to the standard approach, we will be interested in events when no photons were registered. The joint probability of no-count events simultaneously in both the detectors is:

$$
\begin{aligned}
Q_{a b}(\alpha, \beta) & =\left\langle\Psi\left|\hat{Q}_{a}(\alpha) \otimes \hat{Q}_{b}(\beta)\right| \Psi\right\rangle \\
& =\frac{1}{2}|\alpha-\beta|^{2} e^{-|\alpha|^{2}-|\beta|^{2}}
\end{aligned}
$$

where $\alpha$ and $\beta$ are coherent displacements for the modes $a$ and $b$, respectively. The probabilities on single detectors are:

$$
\begin{aligned}
Q_{a}(\alpha) & =\left\langle\Psi\left|\hat{Q}_{a}(\alpha) \otimes \hat{\mathbb{1}}_{b}\right| \Psi\right\rangle=\frac{1}{2}\left(|\alpha|^{2}+1\right) e^{-|\alpha|^{2}}, \\
Q_{b}(\beta) & =\left\langle\Psi\left|\hat{\mathbb{1}}_{a} \otimes \hat{Q}_{b}(\beta)\right| \Psi\right\rangle=\frac{1}{2}\left(|\beta|^{2}+1\right) e^{-|\beta|^{2}} .
\end{aligned}
$$

The measurement is now performed for two settings of the coherent displacement in each of the apparatuses: either zero, or $\alpha$ for the mode $a$ and $\beta$ for the mode $b$. From the resulting four different correlation functions we build the Clauser-Horne combination [9]:

$$
\begin{aligned}
\mathcal{C H}= & Q_{a}(0)+Q_{b}(0)-Q_{a b}(0,0) \\
& -Q_{a b}(\alpha, 0)-Q_{a b}(0, \beta)+Q_{a b}(\alpha, \beta),
\end{aligned}
$$

which for local theories satisfies the inequality $0 \leq \mathcal{C H} \leq$ 1. We will take the coherent displacements to have equal magnitudes $|\alpha|^{2}=|\beta|^{2}=\mathcal{J}$ and an arbitrary phase difference $\beta=e^{2 i \varphi} \alpha$. For these values we obtain

$$
\mathcal{C H}=1-\mathcal{J} e^{-\mathcal{J}}+2 \mathcal{J} e^{-2 \mathcal{J}} \sin ^{2} \varphi .
$$

As depicted in Fig. 2, this result violates the upper bound imposed by local theories. The violation is most significant for the phase $\varphi$ which maximizes the last term in Eq. (7). This takes place when the coherent displacements have opposite phases $\beta=-\alpha$.
Let us note that when no displacements are applied, the detectors measure the bare state $|\Psi\rangle$. This state contains a single photon in the sense that it is an eigenstate of the total photon number operator $\hat{n}_{a}+\hat{n}_{b}$ with an eigenvalue 1 . In this case $Q_{a b}(0,0)=0$, which means that the photon is always registered by one of the detectors.

The only measurement that is required to demonstrate the nonlocality of this state requires single and joint registration of no photons. When the state is not shifted, this measurement is described by the projection on the vacuum state $|0\rangle$. Furthermore, application of a coherent displacement $\hat{D}(\alpha)$ is equivalent to the projection on a coherent state $|\alpha\rangle$. And here comes the most striking link of the quantum nonlocality with the phase space quasidistribution. $Q_{a b}(\alpha, \beta)$ is consequently equal, up to a constant $1 / \pi^{2}$, to the joint $Q$-function of the state $|\Psi\rangle$. The operator $\hat{Q}(\alpha)$, defined above, represents a projection on a coherent state $|\alpha\rangle$, and the correlation function is:

$$
Q_{a b}(\alpha, \beta)=|\langle\alpha, \beta \mid \Psi\rangle|^{2},
$$

where $|\alpha, \beta\rangle=|\alpha\rangle_{a} \otimes|\beta\rangle_{b}$. The probabilities of nocount events on single detectors are given by marginal $Q$-functions:

$$
\begin{aligned}
& Q_{a}(\alpha)=\left\langle\alpha\left|\operatorname{Tr}_{b}(|\Psi\rangle\langle\Psi|)\right| \alpha\right\rangle_{a}, \\
& Q_{b}(\beta)=\left\langle\beta\left|\operatorname{Tr}_{a}(|\Psi\rangle\langle\Psi|)\right| \beta\right\rangle_{b} .
\end{aligned}
$$

Thus, we now clearly see that the $Q$-function contains direct information on nonlocal quantum correlations. If a four-point combination of the type given in Eq. (6) violates the inequality $0 \leq \mathcal{C H} \leq 1$, this immediately certifies the nonlocal properties of the quantum state. This definition has an obvious operational meaning, as we have discussed an experiment in which the nonlocal character of the $Q$-function can be readily tested.

Following a more traditional approach, the combination $\mathcal{C H}$ defined in Eq. (6) can be also related to the probabilities of registering photons by the detectors. A simple calculation shows that $\mathcal{C H}$ can also be expressed as:

$$
\begin{aligned}
\mathcal{C H}= & P_{a}(0)+P_{b}(0)-P_{a b}(0,0) \\
& -P_{a b}(\alpha, 0)-P_{a b}(0, \beta)+P_{a b}(\alpha, \beta)
\end{aligned}
$$

where $P_{a}(\alpha), P_{b}(\beta)$, and $P_{a b}(\alpha, \beta)$ are given by the expectation values over the state $|\Psi\rangle$ of the operators: $\hat{P}_{a}(\alpha)$, $\hat{P}_{b}(\beta)$, and $\hat{P}_{a}(\alpha) \otimes \hat{P}_{b}(\beta)$, respectively.

In order to give an operational meaning to the Wigner function, we will now consider the case when the detectors are capable of resolving the number of absorbed photons. Let us assign to each event +1 or -1 , depending on whether an even or an odd number of photons has been registered. This measurement is described by a pair of projection operators: 


$$
\begin{aligned}
& \hat{\Pi}^{(+)}(\alpha)=\hat{D}(\alpha) \sum_{k=0}^{\infty}|2 k\rangle\langle 2 k| \hat{D}^{\dagger}(\alpha) \\
& \hat{\Pi}^{(-)}(\alpha)=\hat{D}(\alpha) \sum_{k=0}^{\infty}|2 k+1\rangle\langle 2 k+1| \hat{D}^{\dagger}(\alpha) .
\end{aligned}
$$

Using these projections, we construct the correlation function between the outcomes of the apparatuses $a$ and $b$. This correlation has a clear analogy to spin or to photon polarization joint measurements and it is given by the expectation value of the operator:

$$
\hat{\Pi}_{a b}(\alpha, \beta)=\left(\hat{\Pi}_{a}^{(+)}(\alpha)-\hat{\Pi}_{a}^{(-)}(\alpha)\right) \otimes\left(\hat{\Pi}_{b}^{(+)}(\beta)-\hat{\Pi}_{b}^{(-)}(\beta)\right) .
$$

This quantity is proportional to the joint two-mode Wigner function of the state $|\Psi\rangle$. This link becomes obvious if we rewrite $\hat{\Pi}_{a b}(\alpha, \beta)$ to the form:

$$
\hat{\Pi}_{a b}(\alpha, \beta)=\hat{D}_{a}(\alpha) \hat{D}_{b}(\beta)(-1)^{\hat{n}_{a}+\hat{n}_{b}} \hat{D}_{a}^{\dagger}(\alpha) \hat{D}_{b}^{\dagger}(\beta)
$$

showing that the correlation function is given by the displaced parity operator $(-1)^{\hat{n}_{a}+\hat{n}_{b}}$, which is one of equivalent definitions of the Wigner function [10]. It is a striking result, that the nonlocality in a dichotomous correlation measurement in our setup is given directly by the phase space Wigner function of the state $|\Psi\rangle$.

An easy calculation yields the expectation value of the operator $\hat{\Pi}_{a b}(\alpha, \beta)$ over the state $|\Psi\rangle$ :

$$
\begin{aligned}
\Pi_{a b}(\alpha, \beta) & =\left\langle\Psi\left|\hat{\Pi}_{a b}(\alpha, \beta)\right| \Psi\right\rangle \\
& =\left(2|\alpha-\beta|^{2}-1\right) e^{-2|\alpha|^{2}-2|\beta|^{2}} .
\end{aligned}
$$

Now we consider the combination [11]:

$$
\mathcal{B}=\Pi_{a b}(0,0)+\Pi_{a b}(\alpha, 0)+\Pi_{a b}(0, \beta)-\Pi_{a b}(\alpha, \beta)
$$

for which local theories impose the bound $-2 \leq \mathcal{B} \leq 2$. Again we will take equal magnitudes of the coherent displacements $|\alpha|^{2}=|\beta|^{2}=\mathcal{J}$ and a certain phase difference between them $\beta=e^{2 i \varphi} \alpha$. Then the combination $\mathcal{B}$ takes the form:

$$
\mathcal{B}=-1+(4 \mathcal{J}-2) e^{-2 \mathcal{J}}-\left(8 \mathcal{J} \sin ^{2} \varphi-1\right) e^{-4 \mathcal{J}},
$$

which, as shown in Fig. 3, for sufficiently small intensities $\mathcal{J}$ violates the lower bound of the inequality imposed by local theories. As before, the strongest violation is obtained for $\varphi=\pi / 2$, i.e., when the coherent displacements have opposite phases.

It is now an interesting question whether the nonlocality of the Wigner function exhibited in the proposed experiment is connected to its nonpositivity. The Wigner function of the state $|\Psi\rangle$, containing only one photon, is not positive and exhibits the nonlocal character of quantum correlations. The nonlocal character of this phase space function is directly measured in an experiment involving a detection that resolves the number of absorbed photons. However, it should be pointed out that the above measurement for an incoherent mixtures of the two components forming the state $|\Psi\rangle$ leads to a joint correlation equal to $\left(2|\alpha|^{2}+2|\beta|^{2}-1\right) e^{-2|\alpha|^{2}-2|\beta|^{2}}$. Note that this joint correlation is the Wigner function of the incoherent mixture. This function is nonpositive, but it does not exhibit any quantum interference effects and as a result the Bell inequality is not violated in this case. This shows, that the nonpositivity of the Wigner function does not automatically guarantee violation of local realism 12].

In conclusion, we have demonstrated that phase space quasidistribution functions: the Wigner function and the $Q$-function carry explicit information on nonlocality of entangled quantum states. This is due to the fact that these two quasidistributions directly correspond to nonlocal correlation functions which can be measured in a class of photon counting experiments involving application of coherent displacements.

Acknowledgements. This research was partially supported by the Polish KBN grants and by Stypendium Krajowe dla Młodych Naukowców Fundacji na rzecz Nauki Polskiej.

* Also at the Center of Advanced Studies and Department of Physics, University of New Mexico, Albuquerque NM 87131, USA.

[1] E. P. Wigner, Phys. Rev. 40, 749 (1932).

[2] For a review see M. Hillery, R. F. O'Connell, M. O. Scully, and E. P. Wigner, Phys. Rep. 106, 121 (1984); W. P. Schleich, E. Mayr, D. Krämer, Quantum Optics in Phase Space (Wiley-VCH, Berlin, 1998).

[3] A. Einstein, B. Podolsky, and N. Rosen, Phys. Rev. 47, 777 (1935).

[4] J. S. Bell, Physics 1, 195 (1965).

[5] A. Peres, Quantum theory: Concepts and Methods, (Kluwer Academic Publishers, 1993, Dordrecht, The Netherlands).

[6] J. S. Bell, Speakable and unspeakable in quantum mechanics (Cambridge University Press, Cambridge, 1987), Chap. 21; A. M. Cetto, L. De La Pena, and E. Santos, Phys. Lett. A113, 304 (1985); L. M. Johansen, Phys. Lett. A236, 173 (1997); O. Cohen, Phys. Rev. A 56, 3484 (1997).

[7] S. M. Tan, D. F. Walls, and M. J. Collett, Phys. Rev. Lett. 66, 252 (1991); L. Hardy, Phys. Rev. Lett. 73, 2279 (1994).

[8] H. M. Wiseman and G. J. Milburn, Phys. Rev. A 70, 548 (1993); K. Banaszek and K. Wódkiewicz, Phys. Rev. Lett. 76, 4344 (1996); S. Wallentowitz and W. Vogel, Phys. Rev. A 53, 4528 (1996). 
[9] J. F. Clauser and M. A Horne, Phys. Rev. D 10, 526 (1974).

[10] A. Royer, Phys. Rev. A 15, 449 (1977); H. Moya-Cessa and P. L. Knight, Phys. Rev. A 48, 2479 (1993).

[11] J. F. Clauser, M. A. Horne, A. Shimony, and R. A Holt, Phys. Rev. Lett. 23, 880 (1969); J. S. Bell, in Foundations of Quantum Mechanics, ed. by B. d'Espagnat (New York, Academic, 1971).

[12] This and other issues related to the nonpositivity and the nonlocality of the Wigner function will be addressed in a separate publication.

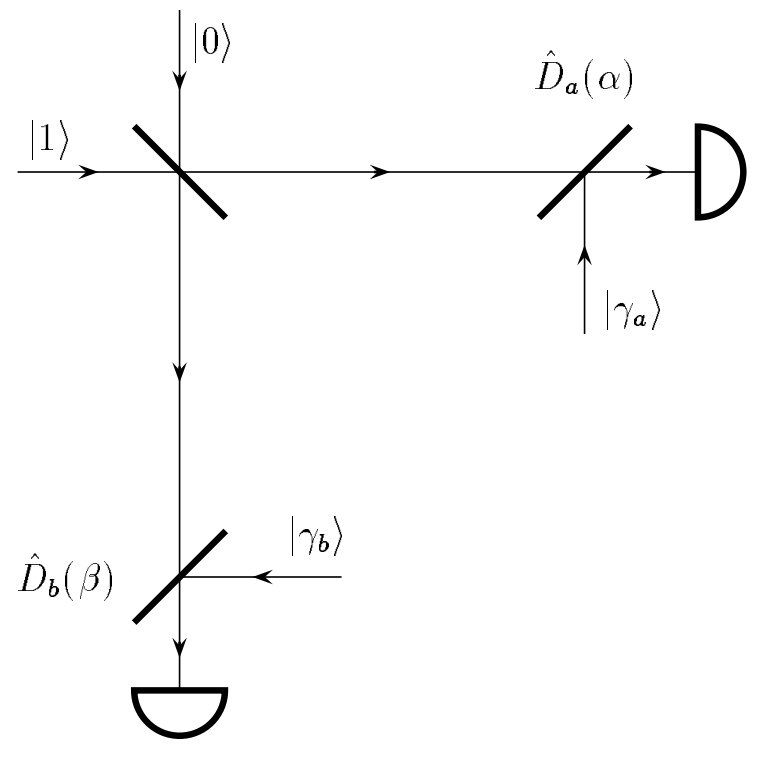

FIG. 1. The optical setup proposed to demonstrate quantum nonlocality in phase space. A single photon incident on a 50:50 beam splitter generates a quantum singlet-like state. The measuring devices are photon counting detectors preceded by beam splitters. The beam splitters have the transmission coefficient close to one, and strong coherent states injected into the auxiliary ports. In this limit, they effectively perform coherent displacements $\hat{D}_{a}(\alpha)$ and $\hat{D}_{b}(\beta)$ on the two modes of the input field.

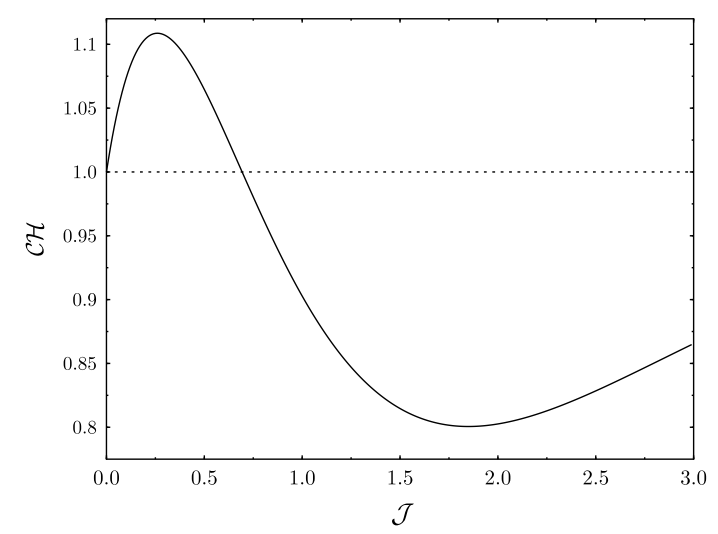

FIG. 2. The plot of the Clauser-Horne combination defined in Eq. (6) as a function of the intensity of coherent displacements $\mathcal{J}=|\alpha|^{2}=|\beta|^{2}$, for opposite phases $\beta=-\alpha$. The dotted line indicates the upper bound imposed by local theories.

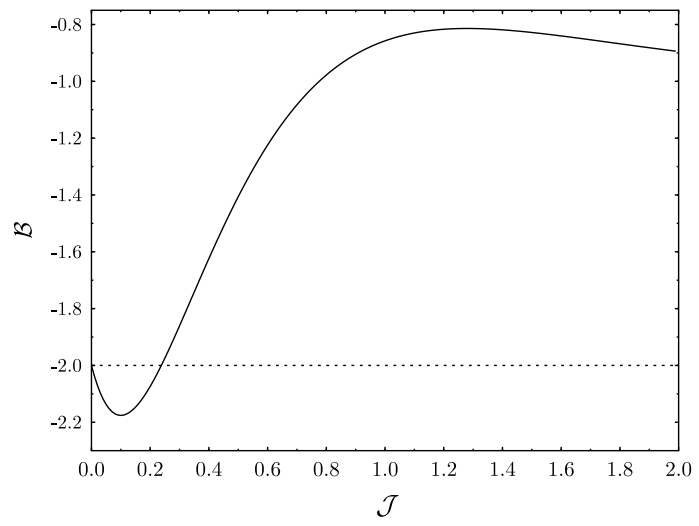

FIG. 3. The plot of the combination defined in Eq. (16) as a function of the magnitude of coherent displacements parameterized with $\mathcal{J}=|\alpha|^{2}=|\beta|^{2}$, for $\beta=-\alpha$. The dotted line indicates the lower bound imposed by local theories. 